\title{
Novel method of knotless vesicourethral anastomosis during robot-assisted radical prostatectomy: feasibility study and early outcomes in 30 patients using the interlocked barbed unidirectional V-LOC180 suture
}

\author{
Kevin C. Zorn, MD, FRCSC; Hugues Widmer, MD; Jean-Baptiste Lattouf, MD; Dan Liberman, MD; \\ Naeem Bhojani, MD; Quoc-Dien Trinh, MD; Maxine Sun, MD; Pierre I. Karakiewicz, MD, FRCSC; \\ Ronald Denis, MD; Assaad El-Hakim, MD, FRCSC
}

Section of Urology, Department of Surgery, University of Montreal, Sacre-Coeur de Montreal Hospital, Montreal, QC

Cite as: Can Urol Assoc J 2011;5(3):188-94; D01:10.5489/cuaj.10194

\begin{abstract}
Purpose: Our purpose was to describe the safety and feasibility of a running posterior reconstruction (PR) integrated with continuous vesicourethral anastomosis (VUA) using a novel self-cinching unidirectional barbed suture in robot-assisted radical prostatectomy (RARP).

Methods: Between March and October 2010, 30 consecutive patients with organ-confined prostate cancer underwent RARP by an experienced single surgeon (KCZ). Upon completion of radical prostatectomy, urinary reconstruction was carried out using 2 knotless, interlocked 6-inches 3-0 V-Loc-180 suture. The left tail of the suture was initially used for PR (starting at 5-o'clock and ran to re-approximate the retrotrigonal layer to the rectourethralis) followed by left-sided VUA (from 6- to 12-o'clock), while the right-sided suture completed the right-sided VUA. Assurance of watertight closure with an intraoperative $300 \mathrm{cc}$ saline visual cystogram was performed in all cases prior to case completion. Perioperative outcomes and 30-day complications were recorded. Results: All anastamoses were performed without assistance and without knot tying. Median time for nurse setup and urinary reconstruction was 40 seconds (interquartile range [IQR] 25-60) and 14.6 min (IQR 10-18), respectively. The need to readjust suture tension or place Lapra-Ty clips (Ethicon Endo-Surgery, Cincinnati, $\mathrm{OH})$ to establish watertight closure was observed in 2 cases $(7 \%)$. No patient had clinical urinary leak and there was no urinary retention after catheter removal on mean postoperative day 5 (IQR 4-6). Conclusions: Our clinical experience with a novel technique using the interlocked V-Loc suture during RARP for both PR and anastomosis appears to be safe and efficient. Using the barbed suture prevents slippage and eliminates the need for bedside assistance to maintain suture tension or knot tying, thus assuring watertight tissue closure.
\end{abstract}

\section{Résumé}

Objectif : Notre but était de décrire l'innocuité et la faisabilité $\mathrm{d}^{\prime}$ une reconstruction postérieure (RP) intégrée à une anastomose vésico-urétrale continue à l'aide de la nouvelle technique de suture avec fils barbelés unidirectionnels et ancrage automatique après prostatectomie radicale assistée par robot (PRAR).

Méthodologie : Entre mars et octobre 2010, 30 patients consécutifs atteints d'un cancer de la prostate confiné à la glande ont subi une PRAR effectuée par un chirurgien expérimenté (KCZ). Après la prostatectomie radicale, une reconstruction urinaire a été entreprise à l'aide de 2 sutures 3-0 de 6 pouces sans noeud par le dispositif V-Loc 180. L'extension gauche de la suture a d'abord été utilisée pour la PR (en commençant à 5 heures et en poursuivant pour rapprocher la couche rétrotrigonale du muscle recto-urétral) et suivie d'une anatostomose vésico-urétrale du côté gauche (de 6 à 12 heures), alors que la partie droite de la suture a permis de terminer l'anastomose vésico-urétrale droite. Une fermeture hermétique par cystogramme visuel intraopératoire avec $300 \mathrm{~mL}$ de solution salée dans tous les cas a été réalisée avant la fin de l'intervention. Les résultats peropératoires et les complications émergeant pendant les 30 jours suivants ont été consignés.

Résultats : Toutes les anastomoses ont été effectuées sans aide et sans nœud. Le temps médian pour la préparation par l'infirmière et la reconstruction urinaire était de 40 secondes (écart interquartile [EIQ] 25-60) et de 14,6 minutes (EIQ 10-18), respectivement. Dans 2 cas $(7 \%)$, on a eu besoin de rajuster la tension des points de suture ou de placer des agrafes LapraTy (Ethicon Endo-Surgery, Cincinnati, $\mathrm{OH}$ ) pour assurer une fermeture hermétique. Aucun patient n'a présenté de fuite urinaire clinique ni aucune rétention urinaire après le retrait du cathéter en moyenne 5 jours après l'opération (EIQ 4-6).

Conclusions : Selon notre expérience clinique, cette nouvelle technique de fermeture de plaie par le dispositif V-Loc pour une PRAR avec anastomose semble sans danger et efficace. L'usage des fils 
barbelés empêche le glissement et élimine le besoin d'aide afin d'assurer la bonne tension des points de suture ou de noeuds, et assure une fermeture hermétique des tissus.

\section{Introduction}

Robot-assisted radical prostatectomy (RARP) continues to gain widespread popularity for the management of localized prostate cancer. Despite the benefits of improved 3D, magnified optics and miniature, endowristed instruments, watertight vesicourethral anastomosis (VUA) remains one of the challenging aspects of this procedure. In addition, urinary reconstruction can be particularly difficult during the initial surgeon's learning curve in obese men, those with previous prostate surgery, large prostate size and patients with deep and narrow pelvises. Consequences to suboptimal VUA may result in urinary leak, ileus, prolonged catheterization, prolonged hospitalization, long-term incontinence and bladder neck contracture.

The Van Velthoven continuous, monofilament suture reconstruction has become the standard of care for RARP. ${ }^{1}$ Numerous modifications have also been described to facilitate VUA, prevent anastomotic leaks and improve continence outcomes. ${ }^{2-7}$ Despite techniques for posterior rhabdosphincter reconstruction (PR), limitations of the running monofilament reconstruction include loss of tension due to suture loosening. Back-slippage of tension is increased by the steep Trandelenburg position that naturally pulls the bladder away from the operative field. As such, most surgeons have adapted their technique by retightening the anastomosis with every throw, using Lapra-Ty (Ethicon EndoSurgery, Cincinnati, $\mathrm{OH}$ ) clips to periodically secure tension ${ }^{8}$ or asking the bedside assistant to follow the suture-line with a laparoscopic needle-driver. An anastomotic technique that is technically simple and highly dependable would therefore be welcomed.

Recently, the field of plastic surgery was introduced to a new concept of barbed suture, which uses novel unidirectional (V-Loc, Covidien, Mansfield, MA) or bidirectional (Quill, Angiotech, Vancouver, BC) barbs to secure tensionfree tissue approximation..$^{9-11}$ Subsequent studies on porcine enteric anastomoses have demonstrated closure equivalence with maxon (monofilament polyglyconate), but with faster anastomosis times. ${ }^{12}$ Since the initial porcine study by Weld and colleagues, which assessed self-anchoring suture (SAS) material for urinary tract reconstruction, two recent publications have emerged assessing barbed suture for RARP VUA demonstrating feasibility and increased efficiency. ${ }^{13,14}$ These small series, however, used separate sutures for PR and VUA. We have modified the use of the V-Loc suture in a loop, interlocked fashion to complete both posterior rhabdosphincter reconstruction along with VUA postulating an easier and more efficient reconstruction for both nurse personnel and the surgical team. Herein, we present our initial series with this novel technique.

\section{Methods}

\section{Patient cohort}

This is a prospective cohort of 30 consecutive patients undergoing RARP at Sacre-Coeur Hospital in Montreal (University of Montreal Hospital Centre, Montreal, Canada) by a single surgeon (KCZ) between March and October 2010. Urinary reconstruction was carried out with the V-Loc180 barbed suture for both PR and VUA. All patients were counselled regarding the use of barbed suture and written consent was obtained.

\section{Suture material}

Following radical prostatectomy completion, two 6-inch 3-0 V-Loc 180 barbed sutures were interlocked via the tail loops by the scrub nurse and introduced for robotic reconstruction. The $\mathrm{V}$-Loc 180 absorbable wound closure device was prepared from a copolymer of glycolic acid and trimethylene carbonate and consisted of a barbed absorbable thread armed with a surgical needle at one end and a loop end effector at the other (Fig. 1). The suture had tensile strength of $80 \%$ after 7 days, $75 \%$ after 14 days, and $65 \%$ after 21 days. ${ }^{14}$ It was fully absorbed by 180 days. The distance between the barbs, which are unidirectional, was 0.025 inches (40 barbs per inch). The barb and loop end effector design allowed for tissue approximation without the need to tie surgical knots, except when the two sutures were tied together at the end of the reconstruction. The unidirectional barbs prohibited suture slippage and also evenly distributed tissue forces at several barbed points, such that tearing and ischemic changes in the suture line were avoided if precise bites are taken.

\section{Surgical technique}

The RARP was performed using our previously described technique. ${ }^{3,8,15}$ After radical prostatectomy specimens were collected in an Endo Catch bag (Covidien, Norwalk, CT), the posterior reconstruction and VUA were performed. The full video detailing the $\mathrm{V}$-Loc suture reconstruction can be seen at http://www.youtube.com/user/DrKevinZorn. In the event of a large bladder neck, bladder neck reconstruction was fashioned with figure of eight, 3-0 Monocryl (Ethicon Endo-Surgery, Cincinnati, $\mathrm{OH}$ ) suture at 3- and 9-o'clock positions. The interlocked V-Loc suture (Fig. 2) was then introduced for robotic reconstruction. Posterior reconstruction was first performed with a first bite taken from the 


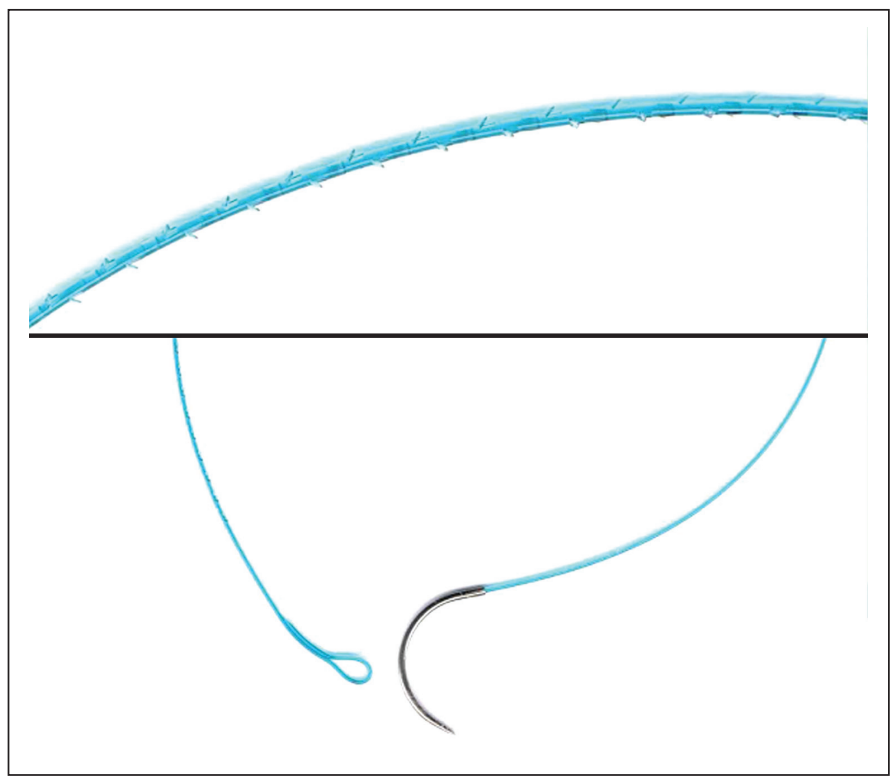

Fig. 1. Close up view of the V-Loc 180 suture demonstrating a) the unidirectional barbs (40 barbs/inch) along the circumference of the suture, b) the terminal loop and c) the tapered V-20 needled. Note the lack of barbs along the last $2 \mathrm{~cm}$ proximal to the needle.

5-o'clock retrotrigonal area followed by periurethral rectourethralis muscle. The suture was pulled through until the interlocked loops abut with the tissue providing resistance, as a knot would. A second bite was then taken from the midline retrotrigonal area behind the bladder followed by a 6-o'clock bite of the periurethral tissue. Care was made to ensure there was no cephalad traction on the bladder prior to cinching. Finally, a final 7-o'clock suture was taken on the bladder-side retrotrigonal tissue again ensuring not to include any mucosa. The left arm of the interlocked V-Loc suture was then lifted cephalad and anteriorly with the left needle driver, while the open right needle driver sat on the bladder tissue to serve as a fulcrum point to avoid tissue tearing. The bladder was thus cinched down with repetitive, short pulls until the bladder neck mucosa was adjacent to the urethral stump with no gap (Fig. 3, part a). This provided for unprecedented hold of auto-tissue tension and allowed the urethral stump to be retracted from the pelvic floor to provide increased length.

Once the PR was complete, the same left arm of the interlocked suture began the VUA starting with a 6-o'clock, out-side-in, transmural bite of the bladder followed by an inside-out bite of the posterior urethra. The assistant may pass the tip of the urethral catheter if there was any question of backwalling the mucosa. The outside-in bites along the bladder and the inside-out urethral bites were continued from 6- to 10- o'clock, each time cinching the tissue with the right needle driver straddling the suture to avoid urethral tearing (Fig. 3, part b). Rather than completing the complete left side of the VUA and therefore obscuring the posterior

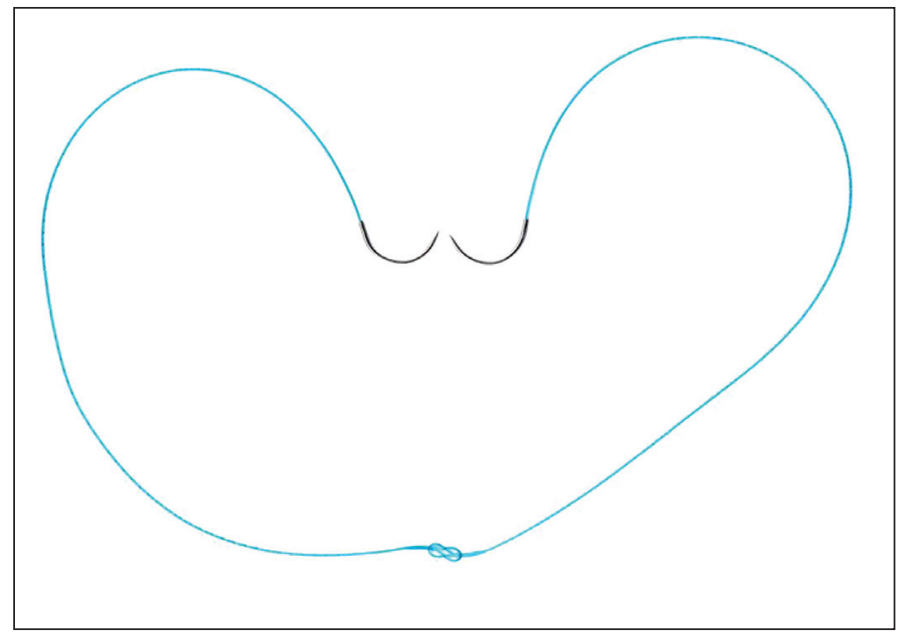

Fig. 2. Interlocked V-Loc configuration for posterior reconstruction and vesicourethral anastomosis. Note the use 2 six-inch sutures in which the loops of both suture are threaded by the opposite needles. As such, an efficient, knotless setup.

anastomosis, the right arm of the V-LOC stitch was used to complete a synchronous process starting from an outside-in 5- o'clock bladder bite to a 5-o'clock inside-out urethral throw. Care was made not to incorporate the neurovascular bundles or rhabdosphincter muscle with aggressive bites. Repetitious passes were continued for the entire right side (5- to 12-o'clock with final pass, each time, independently assuring adequate tension (obviating the need for the bedside assistant to follow tension). The right arm was finally brought through the anterior urethral side and cut with a 2 to $3 \mathrm{~cm}$ stump. The left wall was then completed in a running fashion from the 10- to 12-o'clock location again finishing on the anterior urethra. Prior to cutting the left arm V-Loc suture, the integrity of the VUA was verified with $300 \mathrm{cc}$ normal saline instilled in the bladder (Fig. 3, part c). If any leakage was seen, further cinching of the suture or placement of additional V-Loc bites would be required. The two cut-ends were left untied thus allowing for a completely, knot-free reconstruction. In the event of a large bladder neck, where the last $2 \mathrm{~cm}$ of the $\mathrm{V}$-Loc suture was required (no barbs along this segment), Lapra-Ty clips would help secure tension.

\section{Outcome measures}

Prospective data was collected on 30 consecutive patients for standard perioperative variables. Preoperative, demographic and postoperative data were recorded (Table 1). Intraoperative adverse events (shearing of urethra or bladder neck, need for revision of VUA tension or placement of additional sutures) and postoperative complications (development of postoperative retention following catheter removal on postoperative day 4 to 5, urinoma formation and anastomotic strictures) were recorded. All patients were followed for at least 1 month. Moreover, specific time for V-Loc VUA 
Knotless vesicourethral anastomosis during RP

\begin{tabular}{|c|c|c|}
\hline & Mean & SD \\
\hline Mean age & 59.2 & 6.4 \\
\hline Mean BMI (kg/m²) & 27.7 & 5.2 \\
\hline Mean preoperative PSA (ng/mL) & 5.9 & 3.8 \\
\hline \multicolumn{3}{|l|}{ Clinical stage } \\
\hline cT1c & $20(68 \%)$ & \\
\hline ст2a & $5(16 \%)$ & \\
\hline cT2b & $5(16 \%)$ & \\
\hline \multicolumn{3}{|l|}{ Biopsy Gleason score } \\
\hline 6 & $17(57 \%)$ & \\
\hline 7 & $9(30 \%)$ & \\
\hline$>8$ & $4(13 \%)$ & \\
\hline Mean IPSS & 7.3 & 6.3 \\
\hline Mean SHIM & 2019.9 & 7.3 \\
\hline Previous prostatic surgery (TURP) (\%) & $3(10 \%)$ & \\
\hline Mean prostate weight (g) & 49 & 16.1 \\
\hline
\end{tabular}

suture nurse setup and time to complete watertight VUA were collected. These objective times were recorded by stopwatch during video playback of all cases. The VUA time more specifically was measured from the first PR bite until the left arm V-Loc was cut upon completion of water-tight 300 cc VUA. Continence outcomes were measured with the UCLA Prostate Cancer Index Short Form (UCLA-PCISF) 36v2 validated quality of life questionnaire at 1 and 3 months. ${ }^{16,17}$ Pad-free (no pads/day) was defined as response "no pads" to item 14 (question: how many pads per day?).

\section{Results}

All VUA reconstructions were completely by the console surgeon independently, without any laparoscopic aid from the bed-side assistant. Only occasional catheter tip exposure was necessary upon initiating the posterior floor of the PR and anastomosis. Each throw was secured in place without any loosening of suture loop tension. The mean time for total reconstruction (PR and VUA) was 14.6 minutes (interquartile range [IQR] 10-18) (Table 2). Mean time specifically for posterior reconstruction was 3.3 minutes (IQR 2-5). Bladder neck reconstruction was required in $3(10 \%)$, specifically in 2 of $3(67 \%)$ of men with previous trans-urethral prostate resection (TURP) and 1 with a significantly large, $5-\mathrm{cm}$ median lobe. Mean time for interlocked V-Loc suture setup on the nurse back table was 40 (IQR 25-60) seconds which compared favourably to 302 (IQR 260-470) seconds in our previous 10 cases using conventional monofilament suture, as previously described..$^{8,18}$ Upon completion of the novel V-Loc VUA, 1 (3.3\%) patient was noted to have an anterior

\begin{tabular}{|c|c|c|}
\hline & Mean & SD \\
\hline Total procedure time (min) & 166 & 41.7 \\
\hline Console time (min) & 128 & 30.9 \\
\hline Estimated blood loss $(\mathrm{mL})$ & 167 & 95.4 \\
\hline Urethral tear (\%) & $1(3 \%)$ & \\
\hline Median lobe (\%) & $3(10 \%)$ & \\
\hline Bladder neck reconstruction (\%) & $3(10 \%)$ & \\
\hline \multicolumn{3}{|l|}{ Pathological disease no. (\%) } \\
\hline pT2 & $21(70 \%)$ & \\
\hline рT3 & $9(30 \%)$ & \\
\hline \multicolumn{3}{|l|}{ Positive surgical margins (\%) } \\
\hline Overall & $2(7 \%)$ & \\
\hline pT2 & $1(5 \%)$ & \\
\hline рT3 & $1(11 \%)$ & \\
\hline Length of stay (days) & 1 & 0.3 \\
\hline \multirow[t]{2}{*}{ Catheter duration (days) } & 5 & 1.1 \\
\hline & Mean & IQR \\
\hline Total reconstruction time (min) & 14.6 & $(10-18)$ \\
\hline PR time (min) & 3.3 & $(2-5)$ \\
\hline VUA time (min) & 11.3 & (8-13) \\
\hline Need for further cinching/Lapra-Ty (\%) & $2(7 \%)$ & \\
\hline Nurse back table setup time (seconds) & 40 & $(25-60)$ \\
\hline
\end{tabular}

leak requiring further tightening and 2 additional sutures with the uncut left arm V-Loc suture. Watertightness was verified again with $300 \mathrm{cc}$ prior to case completion. An additional patient required the use of Lapra-Ty clips to secure tension as the complete length of the sutures were needed for urinary reconstruction. This occurred in a post-TURP patient, where despite bladder neck reconstruction prior to anastomosis, had a discrepancy in bladder neck to urethral diameters. Larger paces on the bladder with small travelling distances along the urethral stump lead to an adequate parachuting of tissues for VUA, however, required near all 12 -inches of the 2 interlocked V-Loc sutures. As there were no unidirectional barbs adjacent to the tapered needle, tissue tension was secured with two separate Lapra-Ty clips.

Mean catheter time was 5 days (IQR 4-6). No patient had urinary retention following Foley removal and there was no delayed urinoma formation observed in any of the study patients. With a mean follow-up of 3.3 months, no bladder neck contractures or suture-related complications (irritative voiding symptoms or suture calcification) were observed. The UCLA-PCI-SF36v2 questionnaire $1(47 \%$ vs. $35 \%)$ and 3 month (65\% vs. $51 \%$ ) pad-free continence outcomes were comparable to previous published outcomes. ${ }^{15,19}$ 


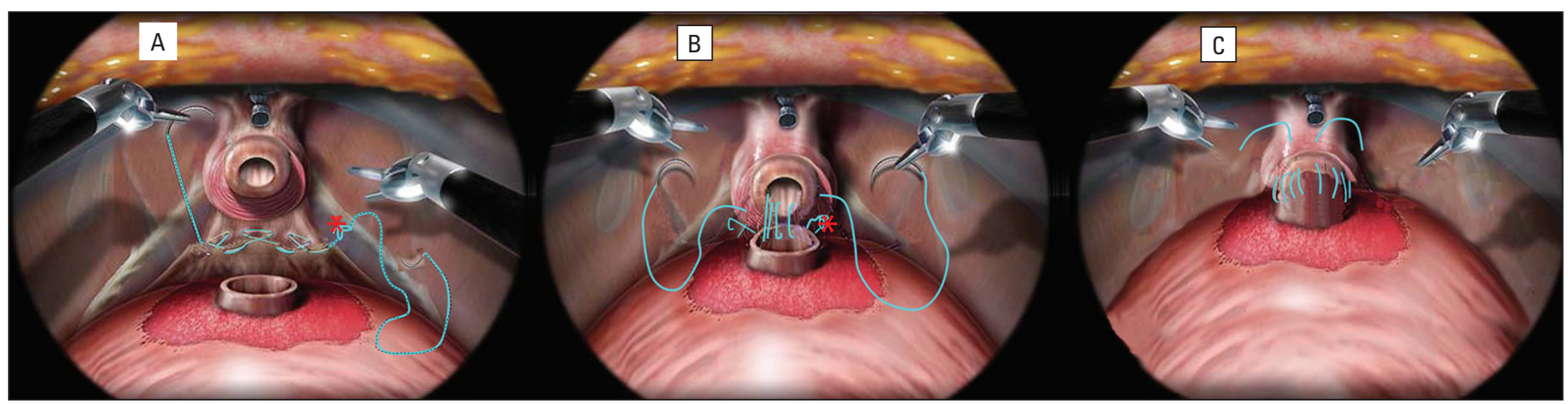

Fig. 3. Overview of the posterior reconstruction (PR) and vesicourethral anastomosis using the interlocked V-LOC suture. a) the left-arm of the hybrid suture is passed initially at the 5-o'clock position of the retrotrigonal tissue. The suture is pulled through until the inter-twined loops oppose the tissue providing resistance. ( $\left(\right.$ red $\left.^{*}\right)$ Using 3 consecutive bites, the PR helps approximate the edge of the bladder neck to the peri-urethral tissue. b) The left arm of the suture is then passed transmurally through the 6-o'clock bladder neck and used to commence a standard Van Velthoven anastomosis. The right arm is then used to close the right-sided VUA. c) A visual cystogram with $300 \mathrm{~mL}$ of saline is performed to ensure no leakage afterwhich the needles are cut and the remaining suture is left in-situ (no knot).

\section{Discussion}

Since its development in 2001, the RARP has been wellreported as safe and effective at tertiary care centres; ${ }^{20,21}$ an increasing proportion of patients undergo RARP for prostate cancer. Unfortunately, in the United States, most RARP cases are performed by low volume surgeons $(<15$ cases/year $) .^{22}$ Higher complications and poorer oncological outcomes have been demonstrated in surgeons with low surgical volumes. Particular to the VUA, surgeons in their early learning experience may only get to this step after several hours on the console, by which time they may be fatigued and result in suboptimal closure.

Most robotic surgeons employ the Van Velthoven anastomosis, ${ }^{1}$ initially described using two 6 -inch monofilament sutures of polyglycolic acid tied together at the ends. The composite suture is used to perform a running anastomosis for efficient and reproducible closure. In their initial experience, VUA times ranged from 14 to 80 minutes. Unfortunately, traditional monofilament suture has a tendency to slip and lose tension. Failure to achieve a watertight closure may result in urinary leakage with increased patient morbidity (prolonged catheterization, ${ }^{23}$ ileus, peritonitis and stricture $^{24}$ ).

Modifications of VUA technique to avoid slippage have been described, including assistant suture holding between bites, readjusting previous bites and the use of Lapra-Ty clips. The use of posterior reconstruction techniques has also been shown to help with reducing anastomotic leakage and potentially improving early urinary continence. ${ }^{3}$ Drawbacks of these techniques, however, include reliance on the assistant, foreign bodies adjacent to the anastomosis, tearing of urethral tissue, suture breakage and increased cost.

In our study, we used two, interlocked 3-0 V-Loc selfretaining sutures to recreate the same 12-inch collective suture described by Van Velthoven. [1] The V-Loc barbed wound closure device, first approved by the FDA in March 2009 , is a unidirectional, self-anchoring barbed suture composed of an absorbable copolymer of trimethylene carbonate and glycolic acid. The tapered surgical needle is at one end, while a self-anchoring loop is at the other. The distance between barbs is 0.025 inches (40 barbs per inch). It is important to be aware that USP designations for diameter are applicable to the V-Loc material prior to barbing. As such, after barb creation, the V-Loc is identified as one size smaller than the non-barbed suture. The V-Loc180 suture has tensile strength of $80 \%, 75 \%$ and $65 \%$ at 7,14 and 21 days, respectively. ${ }^{14}$ Full absorption occurs by day 180 .

Compared to our previous technique using 4-0 Monocryl suture and Lapra-Ty clips, ${ }^{8}$ a significant 8 -fold reduction in nurse setup time (40 vs. 302 seconds), as well as cost $(48.05 \$$ vs. $70.25 \$ C A N)$ was observed. While we feel that it is important for the surgeon to able to adjust suture tension (Lapra-Ty clips), the cost for laparoscopic and robotic surgeons who simply use knot tying, would be $\$ 4.02$ (2 Monocryl sutures). No impact was noted on short-term urinary continence. Moreover, use of the inherent self-retaining suture obviated the need for bedside assistance or knot tying. Such a reduction in instrument clashing and suture entanglement may be most apparent to the inexperienced robotic surgeon. Similar to the outcomes noted by others using barbed VUA suture, we observed a more time-efficient reconstruction. Kaul and colleagues first reported the first clinical use of V-Loc in 51 consecutive RARP patients for VUA. [13] Median time for dual-layer anastomosis was 14 minutes with no leaks on cystography 1 week after surgery (mean Foley time of 7.25 days). What is noteworthy is that their technique uses a third, single barbed 2-0 suture to approximate Denonvilliers' fascia and periurethral tissues. Our technique incorporated the PR and VUA using the same interlocked suture and was tested with $300 \mathrm{~mL}$ (compared to $240 \mathrm{~mL}$ ). With a mean Foley time and follow-up of 
5 days and 3.3 months, respectively, we did not observe any suture-related complications. Tewari and colleagues recently published a retrospective comparative series in which $50 \mathrm{~V}$-Loc barbed VUA cases were compared to 50 conservative anastamoses by the same surgeon. In their analysis using two separate V-Loc sutures and an end knot tying of both suture stumps, the V-Loc group had significantly shorter total reconstruction times (8 vs. $13.5 \mathrm{~min} ; p<0.001$ ). The authors did not state whether intraoperative VUA challenge was performed; since time was measured for only suture reconstruction, this may account for the shorter reconstruction times when compared to our series. Similar to our series, routine radiological cystograms were not performed. Our leak rate of $0 \%$ and the $2 \%$ in the comparative series by Tewari and colleagues compare favourably to urine leaks ranges from $4.5 \%$ to $7.5 \%$ at high-volume RARP centres. ${ }^{20}$ Given the significant reduction by $30 \%$ to $40 \%$ in reconstruction times by experienced robotic surgeons, one can only postulate the greater impact on more novice RARP surgeons; this is especially true for a medical act with such a considerable impact on the short- and long-term functional outcomes of the patient.

There is another bidirectional barbed, double-armed suture (Quill) which is commercially available. In our review, there have been 2 Quill VUA studies demonstrating concerning findings. Weld and colleagues noted significantly higher lamina propria fibrosis and perianastomotic fat fibrosis, but no differences in muscle fibrosis and granulation tissue with the polydiaxonone barbed sutures (Quill) compared with polyglactin (Vicryl, Ethicon, New Brunswick, NJ) sutures when used for bladder neck anastomosis in pigs. ${ }^{13,25}$ In a randomized controlled study comparing monofilament to Quill VUA in 81 men, Williams and colleagues reported that while the barbed polyglyconate sutures were associated with shorter mean anastomosis times (9.7 vs. $9.8 \mathrm{~min}-$ utes; $p=0.014)$, they were associated with more frequent cystogram extravasation 8 days postoperatively $(20.0 \%$ vs. $2.8 \% ; p=0.019)$, longer mean catheterization times (11.1 vs. 8.3 days; $p=0.048) .{ }^{26}$ Such results may be due to the fact that the Quill suture utilizes a cutting rather than a tapered needle, is smaller in diameter when compared to the size-matched V-Loc and has less barbs (28 barbs/inch).

Some of the suggested drawbacks to barbed suture may be the concern for increased adjacent tissue inflammation. However this phenomenon has not been demonstrated in the literature, $9,10,27$ likely as a result of the improved quality, secured, tension-free anastomosis. No long-term data, however, have been reported on reconstruction in the urinary tract, only that of plastic soft tissue reconstruction. Second, the concept of difficulty withdrawing back the suture from tissue was not observed in our series. In 2 cases, the needle had incorporated a small amount of the catheter and was continued to be used for 1 to 2 other tissue bites before being recognized. The robotic needle drivers were easily able to withdraw the V-Loc suture without trauma or fracture.

Despite its merit, there are several limitations of our study that are worthy of mention, namely the small, non-randomized single-surgeon cohort and short-term follow-up. We are currently performing our randomized, controlled study to better scientifically evaluate whether the improved surgical times will translate into improved patient outcomes, namely in continence, erectile function, catheter removal times and oncological results. Similarly, in a Canadian environment with only 11 available robotic centres, we are evaluating the effectiveness of the V-Loc suture in our pure laparoscopic radical prostatectomy cases.

\section{Conclusion}

Our series further demonstrates the safety effectiveness of barbed, self-retaining V-Loc suture for urinary reconstruction during RARP. Using our novel interlocked, double-arm composite, both posterior reconstruction and anastomosis can be performed assistant-less and without the need for knot tying. The self-retaining barbs help distribute tension throughout the reconstruction helps significantly reduce operative times even in already expert hands. Longer-term follow-up is necessary to evaluate the risk for fibrosis when used for urinary reconstruction.

Competing interests: None declared.

This paper has been peer-reviewed

\section{References}

1. Van Velthoven RF, Ahlering TE, Peltier A, et al. Technique for laparoscopic running urethrovesical anastomosis: The single knot method. Urology 2003;61:699-702.

2. Menon M, Muhletaler F, Campos M, et al. Assessment of early continence after reconstruction of the periprostatic tissues in patients undergoing computer assisted (robotic) prostatectomy: Results of a 2 group parallel randomized control trial. J Urol 2008;180:1018-23.

3. Gautam G, Rocco B, Patel VR, et al. Posterior rhabdosphincter reconstruction during robot-assisted radical prostatectomy: critical analysis of techniques and outcomes. Urology 2010;76:734-41.

4. Rocco F, Carmignani L, Acquati P, et al. Restoration of posterior aspect of rhabdosphincter shortens continence time after radical retropubic prostatectomy. J Urol 2006;175:2201-6.

5. Tewari A, Jhaveri J, Rao S, et al. Total reconstruction of the vesico-vrethral junction. BJU Int 2008; 101:871-7.

6. Kalisvaart JF, Osann KE, Finley DS, et al. Posterior reconstruction and anterior suspension with single anastomotic suture in robot-assisted laparoscopic radical prostatectomy: a simple method to improve early return of continence. J Robot Surg 2009;3:149-53.

7. Patel VR, Coelho RF, Palmer KJ, et al. Periurethral suspension stitch during robot-assisted laparoscopic radical prostatectomy: description of the technique and continence outcomes. Eur Urol 2009;56:472-8.

8. Zorn KC. Robotic radical prostatectomy: assurance of water-fight vesicourethral anastomotic closure with the Lapra-Ty Clip. J Endourol 2008;22:863-6.

9. Murtha AP, Kaplan AL, Paglia MJ, et al. Evaluation of a novel technique for wound closure using a barbed suture. Plast Reconstr Surg 2006;117:1769-80.

10. Warner JP, Gutowski KA. Abdominoplasty with progressive tension closure using a barbed suture technique. Aesthet Surg J 2009;29:221-5. 
Zorn et al.

11. Rosen AD. Use of absorbable running barbed suture and progressive tension technique in abdominoplasty: A novel approach. Plast Reconstr Surg 2010;125:1024-7.

12. Demyttenaere SV, Nau P, Henn M, et al. Barbed suture for gastrointestinal closure: A randomized control trial. Surg Innov 2009;16:237-42.

13. Kaul S, Sammon J, Bhandari A, et al. A novel method of urethrovesical ansatmosis during robot-assisted radical prostatectomy using a unidirectional barbed wound closure device: Feasibilty study and early outcomes in 51 patients. J Endourol 2010;24:1789-93.

14. Tewari AK, Srivastava A, Sooriakumaran P, et al. Use of a novel absorbable barbed plastic surgical suture enables a "self-cinching" technique of vesicourethral anastomosis during robot-assisted prostatectomy and improves anastomotic times. J Endourol 2010;24:1645-50.

15. Zorn KC, Wille MA, Thong AE, et al. Continued improvement of perioperative, pathological and continence outcomes during 700 robot-assisted radical prostatectomies. Can I Urol 2009; 16:4742-9.

16. Litwin MS, Hays RD, Fink A, et al. Quality-of-life outcomes in men treated for localized prostate cancer. JAMA 1995;273:129-35.

17. Litwin MS, Hays RD, Fink A, et al. The UCLA Prostate Cancer Index: development, reliability, and validity of a health-related quality of life measure. Med Care 1998;36: 1002-12.

18. Woo JR, Shikanov S, Zorn KC, et al. Impact of posterior rhabdosphincter reconstruction during robotassisted radical prostatectomy: retrospective analysis of time to continence. J Endourol 2009;23:1995-9.

19. Reynolds WS, Shikanov SA, Katz MH, et al. Analysis of continence rates following robot-assisted radical prostatectomy: strict leak-free and pad-free continence. Urology 2010;75:431-6.

20. Novara G, Ficarra V, D'Elia C, et al. Prospective evaluation with standardised criteria for postoperative complications after robotic-assisted laparoscopic radical prostatectomy. Eur Urol 2010;57:363-70.
21. Patel VR, Thaly R, Shah K. Robotic radical prostatectomy: outcomes of 500 cases. BJU Int 2007;99:110912.

22. Budäus L, Sun M, Abdollah F, et al. Impact of surgical experience on in-hospital complication rates in patients undergoing minimally invasive prostatectomy: a population-based study. Ann Surg Oncol 2010 Oct 16 [Epub ahead of print].

23. Krane $L S$, Bhandari $M$, Peabody J0, et al. Impact of percutaneous suprapubic tube drainage on patient discomfort after radical prostatectomy. Eur Urol 2009;56:325-30.

24. Msezane LP, Reynolds WS, Gofrit ON, et al. Bladder neck contracture after robot-assisted laparoscopic radical prostatectomy: evaluation of incidence and risk factors and impact on urinary function. J Endourol 2008:22:97-104

25. Weld $\mathrm{KJ}$, Ames $C D$, Hruby $\mathrm{G}$, et al. Evaluation of a novel knotless self-anchoring suture material for urinary tract reconstruction. Urology 2006;67:1133-7.

26. Williams SB, Alemozaffar $M$, Lei $Y$, et al. Randomized controlled trial of barbed polyglyconate versus polyglactin suture for robot-assisted laparoscopic prostatectomy anastomosis: technique and outcomes. Eur Urol 2010 Aug 2 [Epub ahead of print].

27. Leung JC. Barbed, bi-directional surgical sutures: In Vivo strength and histopathology evaluation. Proceedings of the Society for Biomaterials 29th Annual Meeting. April 30-May 3, 2003, Reno, NV:100.

Correspondence: Dr. Kevin C. Zorn, 235 Rene Levesque, Suite 301, Montreal, QC H2X 1N8; fax: 514-861-3021; kevin.zorn@gmail.com 